\title{
Analysis of Non-steady State Temperature Field of Vehicle Engine Cover
}

\author{
Cai Wei $^{1,2, ~ a, ~ Y a o ~ R u i q i a o ~}{ }^{1, *}$, b and Huang Kunyang ${ }^{1, c}$ \\ ${ }^{1}$ Xi'an Hi-tech Institute, Xi'an, Shaanxi 710025, China \\ ${ }^{2}$ Science and Technology on Elctro-Optic Control Laboratory, Luoyang, Henan 471000, China; \\ acCaiwei_bu@163.com, ${ }^{b} 809683381 @ q q . c o m,{ }^{c}$ hqa208@foxmail.com
}

Keywords: analysis; vehicle engine cover; temperature

\begin{abstract}
For the vehicle from the static state to the moving state, the change of the temperature field at the hood has been analyzed. Taking a domestic civilian car as the research object, the boundary conditions were set based on the experimental data, and the temperature field variation of the hood was calculated by using the ANSYS software. Comparing the results of numerical simulation and experimental results, the two have good consistency in two aspects of temperature distribution and temperature numerical curve. This shows that the numerical simulation method adopted in this paper is reliable and accurate, and can be used to predict the temperature distribution of engine hood at different times.
\end{abstract}

\section{Introduction}

The vehicle engine hood is an important infrared feature position on the upper surface of the vehicle under the working condition of the engine. The study of its temperature field is of great significance for accurate vehicle infrared image simulation and vehicle infrared identification and detection. At present, the research on the temperature field of the surface part of the passenger car is mainly concentrated on the passenger compartment of the vehicle ${ }^{[1]}$. The research on the temperature field of engine hood is scarce at home. Although some documents have involved the temperature field of the engine cover in the process of research ${ }^{[2-5]}$, the temperature field of the engine cover is relatively simple. It is not the focus of the research. Even the temperature of the whole engine cover surface is taken as a fixed value directly ${ }^{[5]}$, which obviously has a certain error with the temperature distribution of the engine cover. Therefore, in this paper, a domestic civil car is taken as the research object. The temperature field of the hood of the engine is simulated in detail in the process of the vehicle from rest to motion.

\section{External Field Measurement Test}

Taking a domestic passenger car as an example, the temperature change of the engine hood during the day from rest to movement is studied. During the test, the layout of the temperature sensor nodes inside and outside the hood is shown in Figures 1 and 2.

The test vehicle enters the running state from the static state at the speed of $15 \mathrm{~km} / \mathrm{h}$ and keeps 
the speed constant. The test site was in the eastern suburb of Xi'an. The test time lasted from 15:45 p.m. to 16:10 p.m. in the afternoon. The meteorological station equipment in the test is shown in Figure 3.

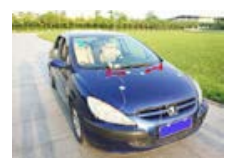

Figure 1. Outer surface node

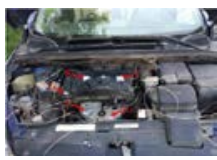

Figure 2. Internal nodes

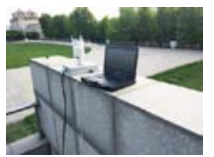

Figure 3. Weather station

\section{Numerical Simulation}

\subsection{Geometric Model}

Building a geometric model based on the real vehicle model 1:1. Considering that the actual vehicle structure is very complex, in order to improve the computing speed and save computing resources, in the modeling process, the parts of the geometric model that have little influence on the temperature field of the engine cover, such as the rear view mirror and the door handle, are ignored. The numerical model of the vehicle hood is shown in Figure 4. Among them, the total number of mesh elements of the engine hood grid is 270526. The flow field model of gas outside the vehicle is shown in Figure 5.

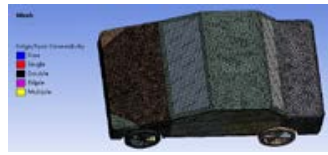

Figure 4. The engine covers external grid schematic diagram

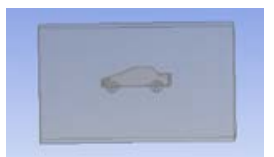

Figure 5. The model of external flow field

\subsection{Parameter Setting of the Model}

The structure of the main components of the engine hood numerical model is measured by the measurement of the vehicle used in the test, as shown in Table 1.

Table 1. The structural parameters of the hood main parts

\begin{tabular}{ccc}
\hline parts & length/(cm) & width/(cm) \\
\hline the hood & 150 & 70 \\
engine & 21 & 43 \\
white part & 13 & 30 \\
\hline
\end{tabular}

The material parameters of the main components of the hood numerical model are shown in table 2.

Table 2. The material parameters of the hood main parts

\begin{tabular}{ccccc}
\hline parts & material & $\begin{array}{c}\text { density } \\
\rho /\left(\mathrm{kg} \cdot \mathrm{m}^{-3}\right)\end{array}$ & $\begin{array}{c}\text { heat } \\
\text { conductivity } \\
\lambda /\left(\mathrm{W} \cdot \mathrm{m}^{-1} \mathrm{~K}^{-1}\right)\end{array}$ & $\begin{array}{c}\text { Specific heat } \\
\text { capacity } \\
\mathrm{c} /\left(\mathrm{J} \cdot \mathrm{kg}^{-1} \mathrm{k}^{-1}\right)\end{array}$ \\
\hline $\begin{array}{c}\text { engine } \\
\text { cover } \\
\text { the hood }\end{array}$ & polyamide & 1050.00 & 0.24 & 1400.00 \\
white part & $\begin{array}{c}\text { struminium } \\
\text { steel }\end{array}$ & 2770.00 & 226 & 875.00 \\
\hline
\end{tabular}

The surface characteristic parameters of the main components of the hood numerical model are 
shown in table 3.

Table 3. The optical characteristic parameters of the hood main parts surface

\begin{tabular}{cccc}
\hline parts & absorptivity & reflectivity & emissivity \\
\hline engine cover & 0.7 & 0.3 & 0.95 \\
interal surface of the hood & 0.83 & 0.17 & 0.92 \\
exteral surface of the hood & 0.8 & 0.2 & 0.95 \\
white part & 0.7 & 0.3 & 0.90 \\
\hline
\end{tabular}

The weather parameters of the main components of the engine hood numerical model are shown in Table 4.

Table 4. Weather conditions for daytime tests

\begin{tabular}{ccc}
\hline time & $\begin{array}{c}\text { ambient } \\
\text { temperature } /\left({ }^{\circ} \mathrm{C}\right)\end{array}$ & wind speed $/(\mathrm{m} / \mathrm{s})$ \\
\hline $15: 45$ & 27.1 & 1.3 \\
$16: 00$ & 27 & 0.5 \\
$16: 10$ & 26.8 & 0.8 \\
\hline
\end{tabular}

\section{The Setting of Boundary Conditions}

\subsection{Setting of Boundary Conditions for Solar Radiation}

Based on the actual test situation, the influence of solar radiation is loaded through the solar calculator in the fluent module. The selected test site is located in the eastern suburb of Xi'an, located in the East 7 area. The longitude is 108.4 degrees east and the latitude is 34.1 degrees north latitude. The vehicle runs from east to west. The test date is April 26, 2018, the time is at 15:45 p.m. to the end of the afternoon at 16:10. The gas transmission rate is 0.8 .

\subsection{Setting of Fluid Boundary Conditions Radiation}

In the numerical simulation of two way flow thermal coupling, the fluent module is mainly used to set the relevant parameters of the fluid. Considering the transient problem of the temperature change of the hood, the external gas of the vehicle is bound to be affected by gravity, so the gas is set to an incompressible gas on the basis of the influence of gravity in the numerical simulation, and the absorption coefficient is 0.4.In addition, in the process of vehicle driving, the temperature of the main heat source parts of the vehicle engine and the white parts will change, and there is also heat exchange between the heat source components and the inner surface of the hood. In order to calculate the physical heat transfer in the head of the engine, the corresponding boundary conditions are set up in the transient thermal analysis module based on the measurement data of the temperature measurement module in the test.

\subsection{The Setting of the Inner Boundary Conditions of the Hood}

The temperature data collected at temperatures 1 is shown in figures 6. Temperature data collected by the temperature measuring node 2 is shown in Figure 7. The temperature data collected at temperatures 7 is shown in Figures 8.

Based on the temperature data acquisition as the selection of temperature measurement node 1 sets the white component surface temperature boundary conditions. The temperature data collected 
by the temperature sensor node 7 are selected as the basis for setting the temperature boundary condition of the engine surface.

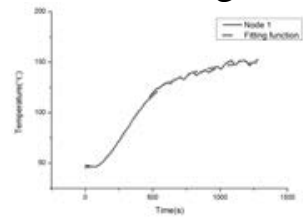

Figure 6 Node 1 temperature data

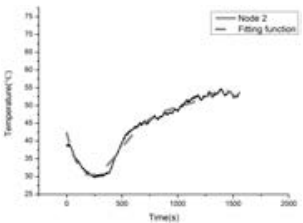

Figure 7 Node 2 temperature data

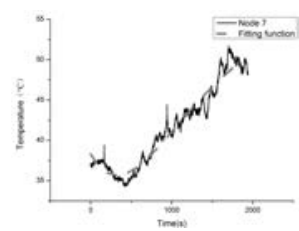

Figure 8 Node 7 temperature data

The function loading method is used in the numerical simulation, which is because in the numerical simulation study, the function loading can reduce the error in the temperature data acquisition during the experiment. The function fitting of the temperature curve is realized by Origin software. The temperature data and function fitting curves of the temperature sensor node 1 , the temperature node 2 and the temperature node 7 are shown in Figures 6, 7 and 8, respectively.

The fitting function of temperature data collected by temperature sensor node 1 is:

$$
y=154.59+(45.725-154.59) /(1+(x / 390.2) \wedge 2.58)
$$

Where $x$ is time of measurement, $y$ is temperature of measurement ,

The fitting function of temperature data collected by temperature sensor node 2 is:

$$
y=42.25-0.125 x+4 e^{-4} x^{2}-3.56 e^{-7} x^{3}-1.246 e^{-11} x^{4}+1.55 e^{-13} x^{5}-5.39 e^{-17} x^{6}
$$

The fitting function of temperature data collected by temperature sensor node 7 is:

$$
y=51.23-0.043 x+8.2 x^{2}-4.7 x^{3}+8.77 x^{4}
$$

\section{Analysis and Verification of Numerical Simulation Results and Experimental Results}

Based on the vehicle's daytime test time, the simulation time is set to 25 minutes. In the transient thermal analysis module of numerical simulation, the results of the temperature distribution on the hood surface are extracted, as shown in Figure 9.Figure 9 is the numerical simulation of the temperature distribution on the outer surface of the vehicle engine cover when the simulation time is 1500 seconds. In the experiment, the infrared gray scale of the temperature distribution on the outer surface of the vehicle hood used by the infrared thermograph at the same time, as shown in Figure 10, is shown in Figure 10.

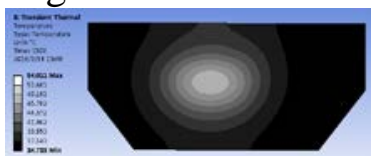

Figure 9 Smulation result

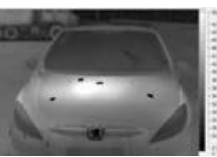

Figure 10 Test result

Comparing with the numerical simulation results of Figure 9 and the test results of Figure 10, it is found that the distribution area of high and low temperature reflected by the two engines is basically the same. The surface temperature distribution of the vehicle engine cover is about 34 degrees -54 degrees in the numerical simulation results. The actual test results show that the actual temperature distribution of the engine cover is about 37 degrees -55 degrees, and the temperature range of the two is basically consistent.

From Figure 11 and Figure 12, it is clear to see that the actual test temperature change curves and the temperature variation curves obtained by the model numerical simulation of the node 4 and the 
node 5 parts of the engine cover surface are not only the same, but the relative error of the temperature values between the two is within $5.3 \%$.

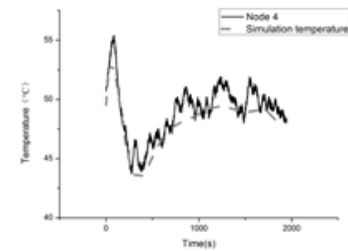

Figure 11 Various temperature variation curves of node 4

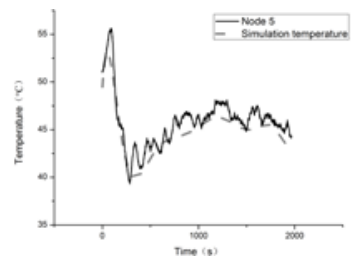

Figure 12 Various temperature variation curves of node 5

\section{Conclusion}

Provides a vehicle during the driving process, numerical simulation method of temperature field in the process of change in non-steady state heat engine condition. The results of this method not only have good accuracy but also can be used to predict the temperature distribution of the engine cover at different time. It is of great significance to the simulation of infrared images in different time periods of the vehicle from rest to motion and the infrared recognition and detection of vehicles.

\section{References}

[1] Zhang Wencan, Chen Jiqing, Lan Fengchong. Research on windshield glazing property effect on vehicle cabin temperature under solar radiation[J]. Journal of Mechanical Engineering, 2011,47(22): 119-125.

[2] Mu Chengpo, Peng Mingsong, Gao Xiang, et al. Infrared image simulation of ground maneuver target and scence [J]. Journal of Beijing Institute of Technology, 2016,25(2): 247-253.

[3] Yu Yanwen, Liu Yijun. Computer modeling and simulation for infrared image of ground objects [J]. Infrared and Laser Engineering, 2008,37(S2): 417-420.

[4] Yu Yanwen, Liu Yijun. Computer simulation of vehicle infrared images [J]. Computer Simulation, 2006,23(12):209-211.

[5] Peng Mingsong. Digital simulation of complicated ground environment and typical targets optical properties [D]. Beijing: Beijing Institute of Technology, 2016. 